\title{
Characteristics, Risk Factors, and Adverse Outcomes of Hyperkalemia in Acute-on-Chronic Liver Failure Patients
}

\author{
Jun-jun Cai $\mathbb{D}^{1},{ }^{1}$ Kai Wang, ${ }^{2,3}$ Hui-qing Jiang $\mathbb{D}^{1},{ }^{1}$ and Tao Han $\mathbb{D}^{4}$ \\ ${ }^{1}$ Department of Gastroenterology, The Second Hospital of Hebei Medical University, Hebei Key Laboratory of Gastroenterology, \\ Hebei Institute of Gastroenterology, Shijiazhuang, China \\ ${ }^{2}$ Dongzhimen Hospital, Beijing University of Chinese Medicine, Beijing, China \\ ${ }^{3}$ Sunsimiao Hospital, Beijing University of Chinese Medicine, Tongchuan, China \\ ${ }^{4}$ Department of Hepatology, Tianjin Third Central Hospital, Tianjin Institute of Hepatobiliary Disease, \\ Tianjin Key Laboratory of Artificial Cell, Tianjin, China
}

Correspondence should be addressed to Hui-qing Jiang; jianghuiqing1959@sina.com and Tao Han; hantaomd@126.com

Received 28 August 2018; Accepted 24 December 2018; Published 27 February 2019

Academic Editor: Hongqun Liu

Copyright @ 2019 Jun-jun Cai et al. This is an open access article distributed under the Creative Commons Attribution License, which permits unrestricted use, distribution, and reproduction in any medium, provided the original work is properly cited.

\begin{abstract}
Background. Hyperkalemia is a serious complication in cirrhotic patients. However, the clinical characteristics, risk factors, and its impact on the outcomes in acute-on-chronic liver failure (ACLF) patients remain unclear. Methods. We retrospectively recruited 650 ACLF patients in this study. The risk factors associated with hyperkalemia and its relationship with 90-day mortality were analyzed using multivariable regression models. Results. Among 650 patients with ACLF, $12.2 \%$ (79/650) had hyperkalemia during hospitalization. Higher admission serum potassium levels and the presence of acute kidney injury (AKI) were independent risk factors for hyperkalemia. The prevalence rates of hyperkalemia in patients with and without AKI were $23.6 \%$ and $4.6 \%$, respectively $(P<0.001)$. Hyperkalemia was a predictor of mortality in AKI and non-AKI patients. The 90 -day mortality rates in non-AKI patients with and without hyperkalemia were $44.4 \%$ and $24.7 \%$, respectively $(P<0.001)$, and in AKI patients with and without hyperkalemia were $80.3 \%$ and $56.6 \%$, respectively $(P<0.001)$. Hepatic encephalopathy $(\mathrm{HE})$, gastrointestinal bleeding, AKI, hyperkalemia, elevated total bilirubin (TBIL) and international normalized ratio (INR) values, and higher Model for End-Stage Liver Disease (MELD) and chronic liver failure-sequential organ failure assessment (CLIF-SOFA) scores were independent risk factors for predicting the 90-day mortality in ACLF patients. Conclusions. Hyperkalemia increases the 90-day mortality in ACLF patients; hyperkalemia is associated with AKI. Patients with both AKI and hyperkalemia had the worst outcome.
\end{abstract}

\section{Introduction}

Impaired potassium homoeostasis represents one of the commonest electrolyte disturbances. Both hypokalemia and hyperkalemia may have immediate deleterious physiological effects and are consistently associated with adverse outcomes [1]. The risk relationship between potassium levels and adverse outcomes is U-shaped, with the lowest risk at serum potassium of 4-4.5 mmol/L $[2,3]$. Patients with advanced cirrhosis frequently have impaired potassium homoeostasis. The prevalence of hyperkalemia in this group of patients is $12 \%-14 \%$, while that in the general population is $2.1 \%-7.0 \%$ [4-6]. Hyperkalemia may lead to arrhythmia, inhibiting the contraction and ventricular fibrillation and even death [7].
Recently, a prospective, multicenter study demonstrated that all cirrhotic patients with a serum potassium concentration of $\geq 4.8 \mathrm{mmol} / \mathrm{L}$ (maximum $5.8 \mathrm{mmol} / \mathrm{L}$ ) died within 1 year [8].

Acute-on-chronic liver failure (ACLF) is a devastating entity characterized by acute deterioration of liver function and one or more extrahepatic organ failures, especially kidney failure $[9,10]$. The kidney plays a major role in potassium homeostasis, with kidney impairment being an especially prominent risk factor for hyperkalemia. A recent study showed that the incidence of hyperkalemia is $65 \%$ higher among children with acute kidney injury (AKI) compared with those without AKI upon admission [11]. Abnormal intake and excretion/loss of potassium resulting from impairment of renal function may lead to hyperkalemia. 


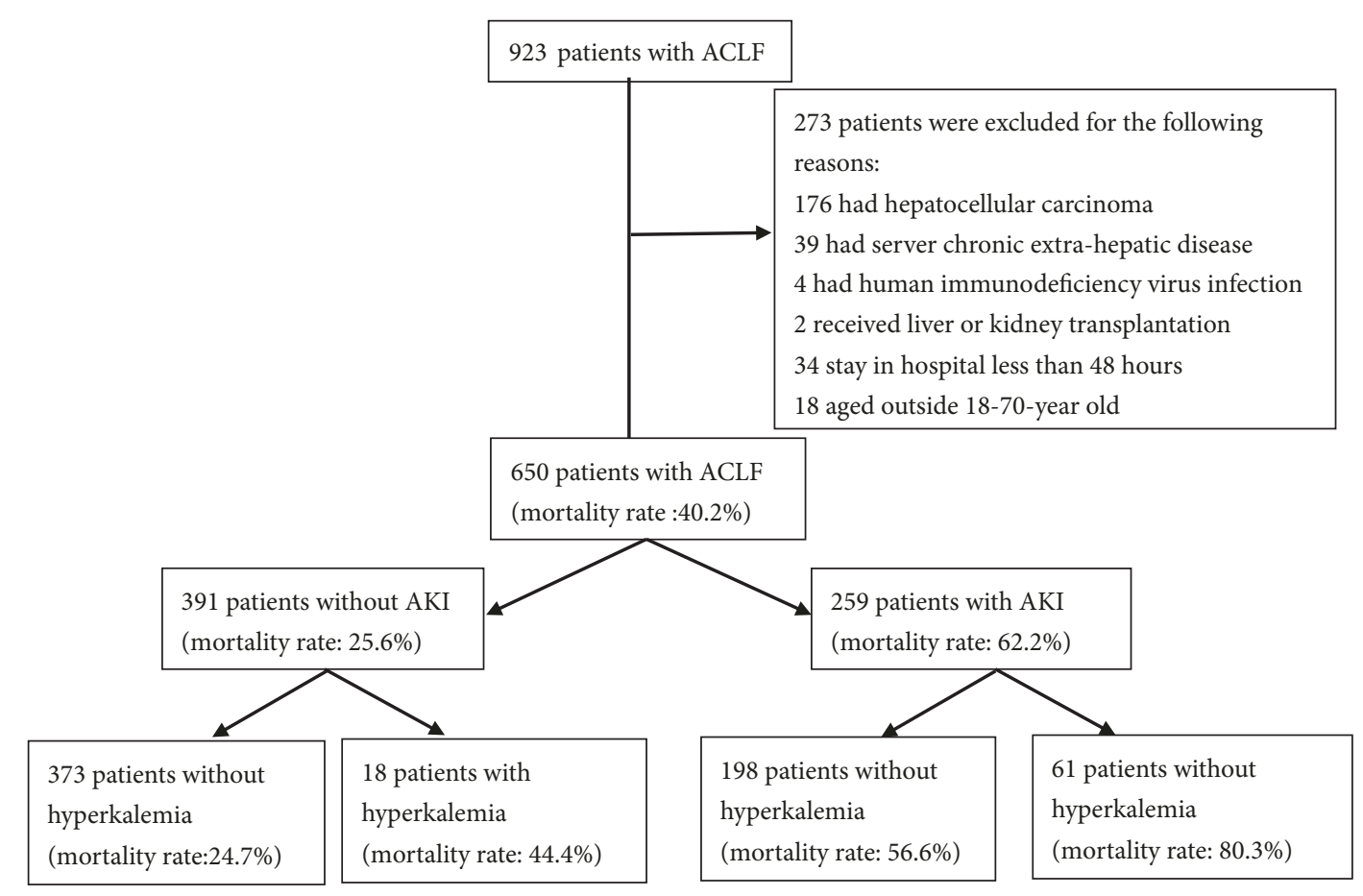

FIGURE 1: Flow diagram of the study groups selection process. ACLF, acute-on-chronic liver failure; AKI, acute kidney injury.

Several hormones, including aldosterone, antidiuretic hormone, are involved in potassium metabolism. Other risk factors of hyperkalemia, in combination with renal impairment, include diabetes, cardiovascular disease, and use of pharmacologic agents that affect the potassium regulation, such as diuretic therapy, potassium supplements, and the renin-angiotensin-aldosterone system (RAAS) blockers [1214]. However, the prevalence, risk factors, and impact of hyperkalemia on the outcome of ACLF patients with or without renal impairment remain poorly defined. Therefore, the present study was to determine the prevalence, risk factors of hyperkalemia, and its impact on the outcome of ACLF patients with or without renal impairment.

\section{Methods}

2.1. Materials and Methods. A flow chart of the patient selection process was presented in Figure 1. We retrospectively recruited 650 patients with ACLF who were hospitalized at Tianjin Third Central Hospital between March 2011 and July 2016. ACLF was defined according to the criteria of Asian Pacific Association for the Study of the Liver (APASL). In brief, acute deterioration of liver function manifested by jaundice (total bilirubin [TBIL]: $\geq 5 \mathrm{mg} / \mathrm{dL}$ or $\geq 85 \mu \mathrm{mol} / \mathrm{L}$ ) and coagulopathy (international normalized ratio [INR] of prothrombin time: $\geq 1.5$ or prothrombin activity [PTA]: $\leq 40 \%]$ ), complicated within 4 weeks with ascites and/or hepatic encephalopathy in a patient with cirrhosis [15]. Exclusion criteria are as follows: (1) patients with hepatocellular carcinoma and nonhepatic neoplasia; (2) immunocompromised patients with human immunodeficiency virus infection; (3) patients with severe chronic extrahepatic disease; (4) patients with a hospital stay of < 48 hours; and (5) patients aged outside 18-70 years old.

Acute kidney injury (AKI) is defined as an increase in serum creatinine value by $0.3 \mathrm{mg} / \mathrm{dL}(26.5 \mu \mathrm{mol} / \mathrm{L})$ within $48 \mathrm{~h}$ or percentage by $\geq 50 \%$ from baseline within 1 week according to The Kidney Disease Improving Global Outcomes (KIDGO) criteria [16]. Hyperkalemia was defined as at least one in-hospital serum potassium level measurement $>5.5 \mathrm{mmol} / \mathrm{L}$. The Model for End-Stage Liver Disease (MELD) score was calculated using the Malinchoc formula [17]. The chronic liver failure-sequential organ failure assessment (CLIF-SOFA) score was calculated according to the criteria of the European Association for the Study of the Liver-Chronic Liver Failure (EASL-CLIF) Consortium [9].

All patients were admitted and received standard supportive treatment. Once recruited, data were collected from the medical records, including patient demographics, vital signs, complete blood count, liver and renal function, INR, electrolytes, and complications of cirrhosis. None of the patients underwent liver transplantation, and none of them were lost to follow-up within the 90-day follow-up period.

2.2. Ethics Statement. The study performed in accordance with the principle of the Declaration of Helsinki (2013 revision) and approved by the Institutional Ethical Committee of Tianjin Third Central Hospital. Written informed consents were obtained during the hospital stay. This trial was registered in the Chinese clinical trial registry: ChiCTR1800017991 (http://www.chictr.org.cn/searchchproj.aspx).

2.3. Statistical Analysis. Normally distributed variables were expressed as mean $\pm \mathrm{SD}$, and nonnormally distributed 


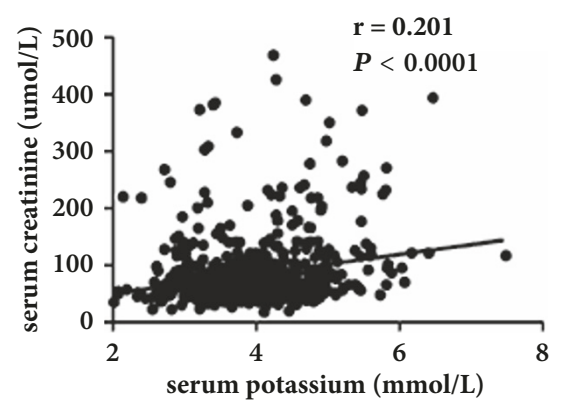

(a)

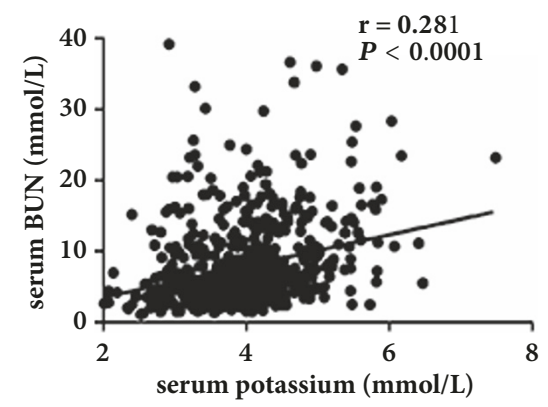

(b)

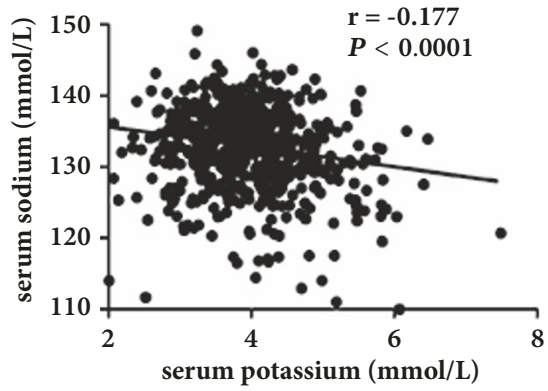

(c)

FIGURE 2: Correlations between serum potassium and serum creatinine, serum BUN, and serum sodium in patients with acute-on-chronic liver failure.

variables were expressed as a median and interquartile range (IQR). Count and percentages were used to describe categorical variables. Two independent groups were compared using the $t$-test for continuous normally distributed variables and the Mann-Whitney $U$ test for nonnormally distributed variables. For categorical variables, comparisons among groups were made using the Chi-squared tests, or Fisher's exact test as appropriate. The Kaplan-Meier method was used to calculate the 90 -day survival probability curves, which were compared with the log-rank test [18]. Multivariate logistic regression analyses were fitted to select the main factors associated with the endpoint. Those found to have a $P$ value of less than 0.05 on univariate analysis were included in the multiple logistic regression models to evaluate the impact on the presence of hyperkalemia and the 90-day mortality of patients. Two-sided $P$ values of $<0.05$ were considered statistically significant. All statistical analyses were performed using SPSS software, version 17.0 (SPSS Inc., Chicago, IL, USA).

\section{Results}

3.1. Characteristics of Patients. Among 650 ACLF patients, 79 (12.2\%) presented hyperkalemia during hospitalization. The characteristics of patients with and without hyperkalemia are summarized in Table 1. Patients with hyperkalemia have a higher baseline serum potassium levels 4.7 (3.75.5) $\mathrm{mmol} / \mathrm{L}$ than those without hyperkalemia 3.9(3.5-4.3) $\mathrm{mmol} / \mathrm{L}(P<0.001)$. Hyperkalemia occurred more frequently in older patients with a higher white blood cell (WBC) count, serum creatinine level, BUN, MELD score, and CLIF-SOFA score $(P<0.001)$ and lower mean arterial pressure, serum sodium and albumin levels $(P<0.05)$. These patients also had higher incidence of hepatic encephalopathy, bacterial infections, acute kidney injury, and gastrointestinal bleeding. Interestingly, no significant differences in the use of potassium-containing preparations, mainly spironolactone, potassium chloride, and potassium citrate, were observed among patients with and without hyperkalemia $(P>0.05)$.

3.2. Correlation of Serum Potassium with Parameters of Renal Function. A positive correlation between serum potassium and both serum creatinine $(\mathrm{r}=0.201, P<0.001)$ and serum
BUN ( $r=0.281, P<0.001)$ was detected in ACLF patients. By contrast, serum potassium had a negative correlation with serum sodium $(\mathrm{r}=-0.177, P<0.001)($ Figure 2$)$.

3.3. Independent Risk Factors Associated with the Presence of Hyperkalemia in ACLF Patients. Multivariate logistic regression analysis showed that independent factors of hyperkalemia were baseline serum potassium levels (odds ratio $[\mathrm{OR}]=3.48,95 \%$ confidence interval $[\mathrm{CI}]=2.309-5.235$, $P<0.001)$ and the presence of acute kidney injury $(\mathrm{OR}=4.77$, 95\% CI $=2.479-9.166$, and $P<0.001)$ (Table 2).

3.4. Relationship between Hyperkalemia and Acute Kidney Injury in ACLF Patients. Hyperkalemia was more prevalent in AKI patients (23.6\%) than in non-AKI patients (4.6\%) $(P<0.001)$. No significant differences in age, use of medications, etiology and complications of cirrhosis, WBC, ALB, INR, and parameters of liver function were observed among AKI patients with and without hyperkalemia $(P>0.05)$. Of note, AKI patients with hyperkalemia had higher serum potassium values and lower TBIL values than AKI patients without hyperkalemia $(P<0.05)$ (Table 3$)$.

3.5. Effects of Hyperkalemia on 90-Day Survival in ACLF Patients. Incidence of 90-day mortality in patients with and without hyperkalemia was $72.2 \%$ and $35.7 \%$, respectively $(P<0.001)$ (Figure 3(a)). In patients with hyperkalemia, nonsurvivors had a higher incidence of hepatic encephalopathy and acute kidney injury than survivors $(P<0.001)$. Moreover, nonsurvivors had a higher INR values, serum potassium concentrations, and MELD scores than survivors $(P<0.05)$ (Table 4).

In patients with hyperkalemia, a multivariate logistic regression analysis was used to evaluate independent factors associated with 90-day mortality. Based on the results of the univariate analysis of parameters presented in Table 4, the independent factors associated with 90-day mortality were high INR values $(\mathrm{OR}=8.19,95 \% \mathrm{CI}=1.668-40.202$, and $P=0.010)$ and acute kidney injury $(\mathrm{OR}=11.36,95 \% \mathrm{CI}=1.838$ 70.186, and $P<0.009$ ) (Table 5(a)).

Due to the close association between hyperkalemia and acute kidney injury, we further stratified the 90-day mortality of patients with hyperkalemia according to the presence 
TABLE 1: Characteristics of all patients according to the presence of hyperkalemia.

\begin{tabular}{|c|c|c|c|}
\hline variables & $\begin{array}{l}\text { without hyperkalemia } \\
\qquad \mathrm{N}=571\end{array}$ & $\begin{array}{l}\text { With hyperkalemia } \\
\qquad \mathrm{N}=79\end{array}$ & $P$-value \\
\hline Age (years) & $51.0 \pm 11.7$ & $53.7 \pm 11.0$ & 0.028 \\
\hline Male-n\% & $436(76.4)$ & $65(82.3)$ & 0.241 \\
\hline Diabetes-n\% & 104(18.2) & $15(19.0)$ & 0.868 \\
\hline Hypertension-n\% & $74(13.0)$ & $13(16.5)$ & 0.392 \\
\hline \multicolumn{4}{|l|}{ Etiology of cirrhosis-n (\%) } \\
\hline Hepatitis B & $327(57.3)$ & $34(43.0)$ & 0.017 \\
\hline Alcoholic & $155(27.1)$ & $35(44.3)$ & 0.002 \\
\hline Hepatitis B plus alcoholic & $30(5.3)$ & $5(6.3)$ & 0.896 \\
\hline Others & $59(10.3)$ & $5(6.3)$ & 0.263 \\
\hline \multicolumn{4}{|l|}{ Medications-n\% } \\
\hline $\begin{array}{l}\text { Diuretic } \\
\text { (frusemide/spironolactone) }\end{array}$ & $493(86.3)$ & $68(86.1)$ & 0.993 \\
\hline Potassium chloride & $263(46.1)$ & $33(41.8)$ & 0.473 \\
\hline Potassium citrate granules & $279(48.9)$ & $34(43.0)$ & 0.332 \\
\hline Beta-blockers & $37(6.5)$ & $5(6.3)$ & 0.959 \\
\hline \multicolumn{4}{|l|}{$\begin{array}{l}\text { Complications of } \\
\text { cirrhosis-n\% }\end{array}$} \\
\hline Ascites & $473(82.8)$ & $67(84.8)$ & 0.661 \\
\hline Bacterial infection & $458(80.2)$ & $74(93.7)$ & 0.004 \\
\hline Hepatic encephalopathy & $173(30.3)$ & $33(41.8)$ & 0.040 \\
\hline Acute kidney injury & $198(34.7)$ & $61(77.2)$ & $<0.001$ \\
\hline Gastrointestinal bleeding & $111(19.4)$ & $28(35.4)$ & 0.001 \\
\hline \multicolumn{4}{|l|}{$\begin{array}{l}\text { Clinical and laboratory } \\
\text { data at admission }\end{array}$} \\
\hline $\mathrm{MAP}(\mathrm{mmHg})$ & $89.6 \pm 12.2$ & $84.7 \pm 15.4$ & 0.001 \\
\hline Heart rate(beats/minute) & $84.2 \pm 14.7$ & $85.0 \pm 16.6$ & 0.658 \\
\hline $\mathrm{WBC}\left(\times 10^{9} / \mathrm{L}\right)$ & $7.7 \pm 4.9$ & $9.9 \pm 6.6$ & $<0.001$ \\
\hline $\operatorname{PLT}\left(\times 10^{9} / \mathrm{L}\right)$ & $78.0(51.0-123.5)$ & $98.5(48.0-130.0)$ & 0.589 \\
\hline $\operatorname{ALB}(g / L)$ & $28.3 \pm 5.2$ & $26.9 \pm 5.8$ & 0.026 \\
\hline $\operatorname{TBIL}(\mu \mathrm{mol} / \mathrm{L})$ & $216.9(130.2-321.2)$ & $144.2(104.5-299.7)$ & 0.128 \\
\hline INR & 2.1(1.7-2.6) & $2.2(1.8-2.9)$ & 0.177 \\
\hline $\mathrm{BUN}(\mathrm{mmol} / \mathrm{L})$ & $5.4(4.0-8.9)$ & $9.6(5.2-14.4)$ & $<0.001$ \\
\hline Serum $\operatorname{Cr}(\mu \mathrm{mol} / \mathrm{L})$ & $61.0(49.0-84.0)$ & $87.0(58.0-122.0)$ & $<0.001$ \\
\hline Serum $\mathrm{Na}^{+}(\mathrm{mmol} / \mathrm{L})$ & $134.1(130.3-137.1)$ & $131.3(125.1-135.6)$ & $<0.001$ \\
\hline Serum $\mathrm{K}^{+}(\mathrm{mmol} / \mathrm{L})$ & $3.9(3.4-4.3)$ & $4.7(3.7-5.5)$ & $<0.001$ \\
\hline CTP score & $12.0(10.0-13.0)$ & $12.0(11.0-13.0)$ & 0.066 \\
\hline MELD score & $21.9 \pm 7.1$ & $25.1 \pm 8.8$ & $<0.001$ \\
\hline CLIF-SOFA score & $7.0(6.0-8.0)$ & $7.0(7.0-11.0)$ & 0.002 \\
\hline Number of deaths-n\% & $204(35.7)$ & $57(72.2)$ & $<0.001$ \\
\hline
\end{tabular}

MAP, mean arterial pressure; WBC, white blood cells; PLT, platelet; ALB, albumin; TBIL, total bilirubin; INR, international normalized ratio; BUN, blood uria nitrogen; Cr, creatinine; CTP, Child-Turcotte-Pugh; MELD, Model for End-Stage Liver Disease; CLIF-SOFA, chronic liver failure-sequential organ failure assessment.

TABLE 2: Risk factors for the presence of hyperkalemia in patients with acute-on-chronic liver failure.

\begin{tabular}{|c|c|c|c|c|c|}
\hline Effect & Estimate & $\mathrm{OR}(95 \% \mathrm{CI})$ & Standard error & Wald X2 & $P$-value \\
\hline Acute kidney injury & 1.562 & $4.77(2.479-9.166)$ & 0.334 & 21.907 & $<0.001$ \\
\hline Admission potassium levels $(\mathrm{mmol} / \mathrm{L})$ & 1.246 & $3.48(2.309-5.235)$ & 0.209 & 35.622 & $<0.001$ \\
\hline
\end{tabular}


TABLE 3: Characteristics of ACLF patients with acute kidney injury according to presence of hyperkalemia.

\begin{tabular}{|c|c|c|c|c|c|}
\hline \multirow{3}{*}{ Variables } & \multicolumn{2}{|c|}{ NO AKI } & \multicolumn{2}{|c|}{ AKI } & \multirow{3}{*}{$P *$-value } \\
\hline & No hyperkalemia & Hyperkalemia & No hyperkalemia & Hyperkalemia & \\
\hline & $\mathrm{N}=373$ & $\mathrm{~N}=18$ & $\mathrm{~N}=198$ & $\mathrm{~N}=61$ & \\
\hline Age (years) & $50.1 \pm 11.7$ & $50.1 \pm 12.7$ & $52.8 \pm 11.5$ & $54.8 \pm 10.3$ & 0.115 \\
\hline Male-n\% & $281(75.3)$ & $15(83.3)$ & $155(78.3)$ & $50(82.0)$ & 1.000 \\
\hline Diabetes-n\% & $63(16.9)$ & $3(16.7)$ & $39(19.7)$ & $11(18.0)$ & 1.000 \\
\hline Hypertension-n\% & $45(12.1)$ & $4(22.2)$ & $30(15.2)$ & $9(14.8)$ & 0.479 \\
\hline \multicolumn{6}{|l|}{ Etiology of cirrhosis-n (\%) } \\
\hline Hepatitis B & $87(23.3)$ & $7(18.8)$ & $83(41.9)$ & $28(45.9)$ & 0.599 \\
\hline Alcoholic & $231(61.9)$ & $11(2.9)$ & $80(40.4)$ & $23(37.7)$ & 0.078 \\
\hline Hepatitis B plus alcoholic & $23(6.2)$ & 0 & $9(4.5)$ & $5(8.2)$ & 0.583 \\
\hline Others & $32(8.6)$ & 0 & $26(13.1)$ & $5(8.2)$ & 0.583 \\
\hline \multicolumn{6}{|l|}{ Medications-n\% } \\
\hline $\begin{array}{l}\text { Diuretic (frusemide } \\
\text { /spironolactone) }\end{array}$ & $309(82.8)$ & $14(77.8)$ & $183(92.4)$ & $55(90.2)$ & 0.324 \\
\hline Potassium chloride & $155(41.6)$ & $5(27.8)$ & $108(54.5)$ & $28(45.9)$ & 0.171 \\
\hline Potassium citrate granules & $184(49.3)$ & $7(38.9)$ & $95(48.0)$ & $27(44.3)$ & 0.686 \\
\hline Beta-blockers & $22(5.9)$ & 0 & $15(7.6)$ & $5(8.2)$ & 0.583 \\
\hline \multicolumn{6}{|l|}{$\begin{array}{l}\text { Complications of } \\
\text { cirrhosis-n\% }\end{array}$} \\
\hline Ascites & $300(80.4)$ & $13(72.2)$ & $173(87.4)$ & $54(88.5)$ & 0.090 \\
\hline Bacterial infection & $273(73.2)$ & $15(83.3)$ & $185(93.4)$ & $59(96.7)$ & 0.075 \\
\hline Hepatic encephalopathy & $93(24.9)$ & $4(22.2)$ & $79(39.9)$ & $29(47.5)$ & 0.063 \\
\hline Gastrointestinal bleeding & $53(14.2)$ & $3(16.7)$ & $58(29.3)$ & $25(41.0)$ & 0.091 \\
\hline \multicolumn{6}{|l|}{$\begin{array}{l}\text { Clinical and laboratory } \\
\text { data at admission }\end{array}$} \\
\hline $\mathrm{MAP}(\mathrm{mmHg})$ & $82.3 \pm 14.6$ & $82.9 \pm 11.2$ & $86.8 \pm 13.9$ & $85.2 \pm 16.5$ & 0.578 \\
\hline Heart rate(beats/minute) & $91.2 \pm 11.0$ & $81.1 \pm 19.4$ & $87.7 \pm 14.4$ & $86.2 \pm 15.7$ & 0.269 \\
\hline $\mathrm{WBC}\left(\times 10^{9} / \mathrm{L}\right)$ & $6.8 \pm 4.0$ & $6.5 \pm 4.1$ & $9.4 \pm 5.8$ & $10.9 \pm 6.8$ & 0.010 \\
\hline $\operatorname{PLT}\left(\times 10^{9} / \mathrm{L}\right)$ & $81.0(51.0-126.0)$ & $75.5(31.8-151.3)$ & $72.5(49.8-115.0)$ & $90.0(55.0-126.0)$ & 0.444 \\
\hline $\operatorname{ALB}(g / L)$ & $29.1 \pm 5.2$ & $28.5 \pm 5.5$ & $26.8 \pm 5.0$ & $26.4 \pm 5.8$ & 0.177 \\
\hline $\operatorname{TBIL}(\mu \mathrm{mol} / \mathrm{L})$ & 210.4(131.3-290.7) & 281.5(106.7-343.6) & $225.5(117.2-360.9)$ & $168.6(104.2-268.3)^{\wedge}$ & 0.086 \\
\hline INR & $2.0(1.7-2.5)$ & $2.3(1.8-3.4)$ & $2.2(1.7-2.9)$ & $2.2(1.8-2.8)$ & 0.532 \\
\hline $\mathrm{BUN}(\mathrm{mmol} / \mathrm{L})$ & $4.8(3.6-6.5)$ & $5.2(3.6-8.4)$ & 8.9(5.1-14.2) & $10.5(6.0-16.2)$ & 0.002 \\
\hline Serum $\operatorname{Cr}(\mu \mathrm{mol} / \mathrm{L})$ & $55.0(46.0-67.0)$ & $63.5(53.8-90.3)$ & $88.0(63.0-139.0)$ & $95.0(67.5-128.0)$ & 0.013 \\
\hline Serum $\mathrm{Na}^{+}(\mathrm{mmol} / \mathrm{L})$ & 134.7(131.7-137.5) & $132.8(135.0-137.0)$ & $132.3(127.9-135.6)$ & $130.4(125.0-135.3)$ & 0.516 \\
\hline Serum $\mathrm{K}^{+}(\mathrm{mmol} / \mathrm{L})$ & $3.9(3.4-4.2)$ & $4.2(3.6-5.6)$ & $3.9(3.4-4.3)$ & $4.8(3.8-5.5)^{\boldsymbol{\Lambda}}$ & 0.599 \\
\hline CTP score & $11.0(10.0-12.0)$ & $12.0(10.0-13.0)$ & $12.0(11.0-13.0)$ & 12.0(11.0-13.0) & 0.398 \\
\hline MELD score & $19.8 \pm 5.7$ & $24.5 \pm 7.6^{\square}$ & $25.9 \pm 8.0$ & $25.2 \pm 9.1$ & 0.767 \\
\hline CLIF-SOFA score & $7.0(6.0-8.0)$ & $7.5(7.0-11.3)^{\square}$ & $8.0(7.0-10.0)$ & $7.0(6.5-11.0)$ & 0.275 \\
\hline
\end{tabular}

$P *$ value between patients with and without AKI with hyperkalemia; $P^{\boldsymbol{\bullet}}<0.05$ patients without AKI no hyperkalemia versus hyperkalemia; $P^{\wedge}<0.05$ patients with AKI no hyperkalemia versus hyperkalemia.

MAP, mean arterial pressure; WBC, white blood cells; PLT, platelet; ALB, albumin; TBIL, total bilirubin; INR, international normalized ratio; BUN, blood urea nitrogen; Cr, creatinine; CTP, Child-Tturcotte-Pugh; MELD, Model for End-Stage Liver Disease; CLIF-SOFA, chronic liver failure-sequential organ failure assessment.

of acute kidney injury. The corresponding survival curves of AKI and non-AKI patients according to incident hyperkalemia are shown in Figure 3(b). The 90-day mortality rates of non-AKI patients with and without hyperkalemia were $44.4 \%$ and $24.7 \%$, respectively $(P<0.001)$. By contrast, the 90-day mortality rates of AKI patients with and without hyperkalemia were $80.3 \%$ and $56.6 \%$, respectively $(P<0.001)$.

A multivariate logistic regression analysis of all ACLF patients showed that HE, gastrointestinal bleeding, AKI and hyperkalemia, elevated TBIL and INR values, high MELD, and CLIF-SOFA scores were independent risk factors for 


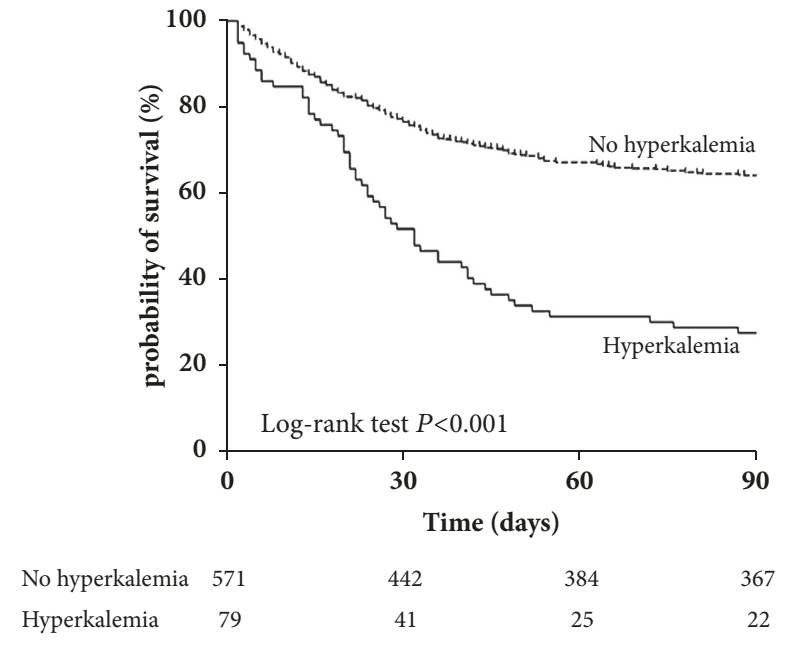

(a)

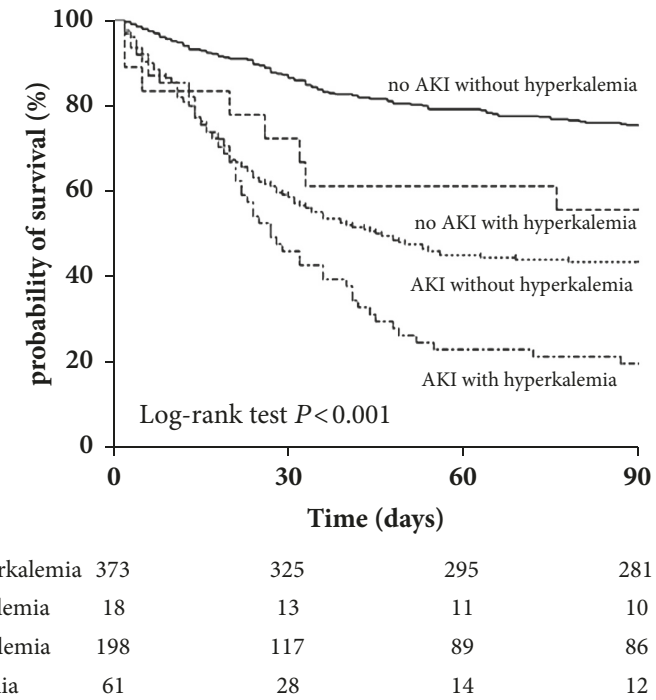

(b)

FIGURE 3: Kaplan-Meier survival curves (a) in patients with and without hyperkalemia (b) in patients with hyperkalemia according to presence of acute kidney injury (AKI).

predicting the 90-day mortality, with ORs of 3.34, 2.00, 2.35, $2.98,1.00,1.77,0.90$, and 1.19 , respectively (Table $5(\mathrm{~b})$ ).

\section{Discussion}

Hyperkalemia is one of the most serious electrolyte disturbances with an associated sudden cardiac death and fatal arrhythmias. Under normal conditions, serum potassium concentration is strictly regulated by redundancy of homeostatic mechanism and maintained within a narrow range of $3.5-5.5 \mathrm{mmol} / \mathrm{L}$. Once these homeostatic mechanisms are disrupted, potassium abnormalities may occur. With the increased in serum potassium concentrations, the risk of adverse outcome increases substantially, which makes hyperkalemia a medical emergency that need special attention. However, in clinical practice, no single threshold could identify the imminent adverse outcome of hyperkalemia in different patients [19].

In our study, 12.2\% ACLF patients developed hyperkalemia during hospitalization. Hyperkalemia commonly affects older patients with a higher WBC count, more severe hepatorenal function, and higher incidence of hepatic encephalopathy, bacterial infections, gastrointestinal bleeding and AKI. Patients with advanced cirrhosis manifest a hyperdynamic circulatory state, which is a progressive vasodilatory syndrome and a key factor in the pathogenesis of various complications of cirrhosis, prominently in the kidney [20]. Vasodilatation leads to a decreased effective arterial blood volume and activation of neurohumoral systems such as RAAS. Increased plasma antidiuretic hormone (ADH) levels lead to water and sodium retention causing ascites and dilution hyponatremia further [4]. In hyponatremia, the $\mathrm{Na}^{+}-\mathrm{K}^{+}$-ATPase pumps sodium ions out of the cell and potassium into the cell [21]. In addition, patients with cirrhosis are characterized by a state of secondary hyperaldosteronism. Aldosterone, a mineralocorticoid hormone, is known to regulate the sodium reabsorption and potassium secretion in renal cortical-collecting duct. Aldosterone acts by increasing the number of open sodium channels, leading to increased sodium reabsorption and potassium secretion. This mechanism explains the negative relationship between serum potassium and serum sodium in ACLF patients.

Gastrointestinal bleeding, increased diuretic doses, large volume paracentesis, and lactulose-induced diarrhea may lead to a state of hypovolemia in ACLF patients. Hypovolemic activates the renin-angiotensin system [21]. The increased circulating aldosterone stimulates renal sodium retention and affects potassium excretion. This perturbed potassium homoeostasis is further disturbed by pharmacologic agents such as antialdosterones and inhibitors of RAAS, which are known to affect serum potassium. Therefore, hyperkalemia can be considered a late event in the natural history of cirrhosis, which occurs after the onset of ascites, gastrointestinal bleeding, and renal impairment [4]. Of note, diuretics such as spironolactone, and various potassium-containing preparations (potassium chloride, potassium citrate, and potassium magnesium aspartate) are commonly used in the management of cirrhotic patients and may lead to hyperkalemia. However, in our study, no significant differences in the use of potassium-saving drugs were observed among patients with and without hyperkalemia $(P>0.05)$.

We also found that higher baseline serum potassium levels and the presence of AKI were the independent risk factors of hyperkalemia. We further analyzed the relationship between hyperkalemia and AKI. We found that hyperkalemia was more frequently seen in patients with AKI. Surprisingly, no significant differences in age, etiology, and complications of cirrhosis and other parameters except for the higher baseline serum potassium concentration were observed among AKI patients with and without hyperkalemia on admission. Hence, it is difficult for clinicians to identify the early presence of hyperkalemia in AKI patients. However, the 90-day 
TABLE 4: Comparison between patients with hyperkalemia who died or survived.

\begin{tabular}{|c|c|c|c|}
\hline Variables & $\begin{array}{c}\text { Survival } \\
\mathrm{N}=22\end{array}$ & $\begin{array}{c}\text { Non-survival } \\
\mathrm{N}=57\end{array}$ & $P$-value \\
\hline Age (years) & $52.5 \pm 7.9$ & $54.2 \pm 12.0$ & 0.563 \\
\hline Male-n\% & $17(77.3)$ & $48(84.2)$ & 0.469 \\
\hline \multicolumn{4}{|l|}{ Etiology of cirrhosis-n (\%) } \\
\hline Hepatitis B & $7(31.8)$ & $23(40.4)$ & 0.484 \\
\hline Alcoholic & $12(54.5)$ & $26(45.6)$ & 0.476 \\
\hline Hepatitis B plus alcoholic & $1(4.5)$ & $4(7.0)$ & 1.000 \\
\hline others & $2(9.1)$ & $3(5.3)$ & 0.614 \\
\hline \multicolumn{4}{|l|}{ Medications-n\% } \\
\hline Diuretic (frusemide/spironolactone) & $20(90.9)$ & $48(84.2)$ & 0.683 \\
\hline Potassium chloride & $11(50.0)$ & $22(38.6)$ & 0.357 \\
\hline Potassium citrate granules & $9(40.9)$ & $25(43.9)$ & 0.812 \\
\hline Beta-blockers & $1(4.5)$ & $4(7.0)$ & 1.000 \\
\hline \multicolumn{4}{|l|}{ Complications of cirrhosis-n\% } \\
\hline Ascites & $17(77.3)$ & $50(87.7)$ & 0.246 \\
\hline Bacterial infection & 20(90.9) & $54(94.7)$ & 0.614 \\
\hline Hepatic encephalopathy & $5(22.7)$ & $28(49.1)$ & 0.033 \\
\hline Acute kidney injury & $12(54.5)$ & $49(86.0)$ & 0.003 \\
\hline Gastrointestinal bleeding & $5(22.7)$ & $23(40.4)$ & 0.142 \\
\hline \multicolumn{4}{|c|}{ Clinical and laboratory data at admission } \\
\hline $\mathrm{MAP}(\mathrm{mmHg})$ & $62.6 \pm 15.7$ & $83.7 \pm 15.9$ & 0.327 \\
\hline Heart rate(beats/minute) & $81.8 \pm 11.7$ & $86.3 \pm 18.0$ & 0.292 \\
\hline $\mathrm{WBC}\left(\times 10^{9} / \mathrm{L}\right)$ & $8.5 \pm 4.8$ & $10.5 \pm 7.1$ & 0.248 \\
\hline $\operatorname{PLT}\left(\times 10^{9} / \mathrm{L}\right)$ & $104.0(72.5-152)$ & $77.0(47.0-120.5)$ & 0.056 \\
\hline $\operatorname{ALB}(g / L)$ & $28.4 \pm 3.7$ & $26.3 \pm 6.3$ & 0.151 \\
\hline $\operatorname{TBIL}(\mu \mathrm{mol} / \mathrm{L})$ & $228.8(104.4-343.6)$ & $184.3(106.6-286.9)$ & 0.314 \\
\hline INR & $1.8(1.6-2.1)$ & $2.5(1.8-3.3)$ & 0.003 \\
\hline $\mathrm{BUN}(\mathrm{mmol} / \mathrm{L})$ & $6.6(4.3-10.8)$ & $10.5(5.6-15.3)$ & 0.057 \\
\hline Serum $\operatorname{Cr}(\mu \mathrm{mol} / \mathrm{L})$ & $70.6(54.0-104.0)$ & $95.0(60.0-129.0)$ & 0.077 \\
\hline Serum $\mathrm{Na}^{+}(\mathrm{mmol} / \mathrm{L})$ & 132.2(124.6-137.1) & $131.0(125.2-135.6)$ & 0.600 \\
\hline Serum $\mathrm{K}^{+}(\mathrm{mmol} / \mathrm{L})$ & $3.8(3.4-5.5)$ & $4.9(4.0-5.6)$ & 0.038 \\
\hline CTP score & $11.5(10.0-13.0)$ & $12.0(11.0-13.0)$ & 0.079 \\
\hline MELD score & $22.1 \pm 4.4$ & $26.2 \pm 9.7$ & 0.016 \\
\hline CLIF-SOFA score & 7.0(7.0-10.0) & $8.0(7.0-11.5)$ & 0.596 \\
\hline
\end{tabular}

MAP, mean arterial pressure; WBC, white blood cells; PLT, platelet; ALB, albumin; TBIL, total bilirubin; INR, international normalized ratio; BUN, blood urea nitrogen; Cr, creatinine; CTP, Child-Turcotte-Pugh; MELD, Model for End-Stage Liver Disease; CLIF-SOFA, chronic liver failure-sequential organ failure assessment.

mortality was significantly higher in AKI patients with hyperkalemia than in non-AKI patients without hyperkalemia. Moreover, hyperkalemia is the independent risk factor for the 90-day mortality of ACLF patients. The physiopathologic association between the hyperkalemia and poor prognosis is complex and remains to be elucidated. The mechanisms that drive this poor prognosis are likely due to the occurrence of a severe disease and the use of therapeutic drugs, which in cases with a renal dysfunction may result in hyperkalemia [22]. This result is in line with our findings, indicating the positive relationship between serum potassium and creatinine as well as BUN, which are known markers of renal dysfunction. Hence, it reminds us that appropriate surveillance regimens and the timely correction of abnormal serum potassium levels and renal function are critical, especially in AKI patients.

This study had limitations. Firstly, this was a retrospective and single center study; the results need to be validated in multicenter, prospective study. Secondly, this study may underestimate the incidence and risk of potassium variations in clinical practice, where laboratory surveillance is frequently less assiduous and regular.

In conclusion, $12.2 \%$ of ACLF patients had hyperkalemia, and a close association between hyperkalemia and AKI was detected in ACLF patients. Hyperkalemia is more frequently seen in patients with AKI. The 90-day mortality rates were clearly different among AKI patients with and without hyperkalemia. AKI patients with hyperkalemia had the worst 
TABLE 5: Independent factors associated with 90-day mortality (a) in patients with hyperkalemia and (b) in all ACLF patients.

(a)

\begin{tabular}{lccccc}
\hline Effect & Estimate & OR (95\%CI) & Standard error & Wald X2 & P-value \\
\hline Acute kidney injury & 2.43 & $11.36(1.838-70.186)$ & 0.929 & 6.837 & 0.009 \\
INR & 2.103 & $8.19(1.668-40.202)$ & 0.812 & 6.707 & 0.010 \\
\hline
\end{tabular}

(b)

\begin{tabular}{|c|c|c|c|c|c|}
\hline Effect & Estimate & OR $(95 \% C I)$ & Standard error & Wald X2 & $P$-value \\
\hline Hepatic encephalopathy & 1.206 & $3.34(2.146-5.195)$ & 0.226 & 28.560 & $<0.001$ \\
\hline Gastrointestinal bleeding & 0.693 & $2.00(1.207-3.315)$ & 0.258 & 7.231 & 0.007 \\
\hline Acute kidney injury & 0.853 & $2.35(1.482-3.714)$ & 0.234 & 13.241 & $<0.001$ \\
\hline Hyperkalemia & 1.091 & $2.98(1.539-5.760)$ & 0.337 & 10.505 & 0.001 \\
\hline TBIL & 0.004 & $1.00(1.002-1,006)$ & 0.001 & 13.893 & $<0.001$ \\
\hline INR & 0.568 & $1.77(1.230-2.532)$ & 0.184 & 9.526 & 0.002 \\
\hline MELD & -0.110 & $0.90(0.826-0.973)$ & 0.042 & 6.888 & 0.009 \\
\hline CLIF-SOFA & 0.176 & $1.19(1.065-1.335)$ & 0.058 & 9.307 & 0.002 \\
\hline
\end{tabular}

INR, international normalized ratio; TBIL, total bilirubin; MELD, Model for End-Stage Liver Disease; CLIF-SOFA, chronic liver failure-sequential organ failure assessment.

outcome. HE, gastrointestinal bleeding, AKI, hyperkalemia, elevated TBIL and INR values, and high MELD and CLIFSOFA scores were independent factors for predicting the 90day mortality in ACLF patients. The dynamic monitoring and timely treatment of abnormal serum potassium levels and renal function are critical for improving the patients' prognosis.

\section{Abbreviations}

ACLF: $\quad$ Acute-on-chronic liver failure

AKI: $\quad$ Acute kidney injury

CTP: $\quad$ Child-turcotte-pugh

MELD: $\quad$ Model for End-Stage Liver Disease

CLIF-SOFA: Chronic liver failure-sequential organ failure assessment

HE: $\quad$ Hepatic encephalopathy

WBC: White blood cells

PLT: $\quad$ Platelet

ALB: $\quad$ Albumin

TBIL: $\quad$ Total bilirubin

BUN: $\quad$ Blood urea nitrogen

Cr: Creatinine

INR: International normalized ratio.

\section{Data Availability}

All data are available on the website of Chinese clinical trial registry within six months after the manuscript published.

\section{Conflicts of Interest}

The authors declared no conflicts of interest.

\section{Acknowledgments}

This work was supported by the National 13th 5-year Plan for Hepatitis Research [Grant no. 2017ZX10203201-007] and
Graduate Innovation Fund of Education Department of Hebei Province [Grant no. CXZZBS2017104].

\section{References}

[1] A. S. Desai, J. Liu, M. A. Pfeffer et al., "Incident Hyperkalemia, Hypokalemia, and Clinical Outcomes During Spironolactone Treatment of Heart Failure With Preserved Ejection Fraction: Analysis of the TOPCAT Trial," Journal of Cardiac Failure, vol. 24, no. 5, pp. 313-320, 2018.

[2] C. P. Kovesdy, K. Matsushita, Y. Sang et al., "Serum potassium and adverse outcomes across the range of kidney function: a CKD Prognosis Consortium meta-analysis," European Heart Journal, vol. 39, no. 17, pp. 1535-1542, 2018.

[3] J. Núñez, A. Bayés-Genís, F. Zannad et al., "Long-Term Potassium Monitoring and Dynamics in Heart Failure and Risk of Mortality," Circulation, vol. 137, no. 13, pp. 1320-1330, 2018.

[4] R. Maiwall, S. Kumar, M. K. Sharma, Z. Wani, M. Ozukum, and S. K. Sarin, "Prevalence and prognostic significance of hyperkalemia in hospitalized patients with cirrhosis," Journal of Gastroenterology and Hepatology, vol. 31, no. 5, pp. 988-994, 2016.

[5] E. Nilsson, A. Gasparini, J. Ärnlöv et al., "Incidence and determinants of hyperkalemia and hypokalemia in a large healthcare system," International Journal of Cardiology, vol. 245, pp. 277-284, 2017.

[6] Y. Chen, A. R. Chang, M. A. McAdams DeMarco et al., "Serum Potassium, Mortality, and Kidney Outcomes in the Atherosclerosis Risk in Communities Study," Mayo Clinic Proceedings, vol. 91, no. 10, pp. 1403-1412, 2016.

[7] A. Goyal, J. A. Spertus, K. Gosch et al., "Serum potassium levels and mortality in acute myocardial infarction," Journal of the American Medical Association, vol. 307, no. 2, pp. 157-164, 2012.

[8] S. Wallerstedt, M. Simrén, S. Wahlin et al., "Moderate hyperkalemia in hospitalized patients with cirrhotic ascites indicates a poor prognosis," Scandinavian Journal of Gastroenterology, vol. 48, no. 3, pp. 358-365, 2013.

[9] R. Moreau, R. Jalan, P. Gines et al., "Acute-on-chronic liver failure is a distinct syndrome that develops in patients with 
acute decompensation of cirrhosis," Gastroenterology, vol. 144, no. 7, pp. 1426.e9-1437.e9, 2013.

[10] P. Angeli, E. Rodríguez, S. Piano et al., "Acute kidney injury and acute-on-chronic liver failure classifications in prognosis assessment of patients with acute decompensation of cirrhosis," Gut, vol. 64, no. 10, pp. 1616-1622, 2015.

[11] F. Chowdhury, P. K. Ghosh, K. M. Shahunja et al., "Hyperkalemia Was an Independent Risk Factor for Death While Under Mechanical Ventilation Among Children Hospitalized With Diarrhea in Bangladesh," Global Pediatric Health, vol. 5, Article ID 2333794X1775400, 2018.

[12] M. Legrand, P.-O. Ludes, Z. Massy et al., "Association between hypo- and hyperkalemia and outcome in acute heart failure patients: the role of medications," Clinical Research in Cardiology, vol. 107, no. 3, pp. 214-221, 2018.

[13] G. Bandak, Y. Sang, A. Gasparini et al., "Hyperkalemia after initiating renin-angiotensin system blockade: The Stockholm Creatinine Measurements (SCREAM) project," Journal of the American Heart Association, vol. 6, no. 7, 2017.

[14] P. H. Pun, B. A. Goldstein, J. A. Gallis, J. P. Middleton, and L. P. Svetkey, "Serum Potassium Levels and Risk of Sudden Cardiac Death Among Patients With Chronic Kidney Disease and Significant Coronary Artery Disease," Kidney International Reports, vol. 2, no. 6, pp. 1122-1131, 2017.

[15] S. K. Sarin, C. K. Kedarisetty, Z. Abbas et al., "Acute-onchronic liver failure: consensus recommendations of the Asian Pacific Association for the Study of the Liver (APASL) 2014," Hepatology International, vol. 8, no. 4, pp. 453-471, 2014.

[16] A. K. I. Kdigo, Kidney Disease Improving Global Outcomes, vol. 1, AKI Work Group: Clinical practice guideline for acute kidney injury, 2012.

[17] A. E. Ruf, W. K. Kremers, L. L. Chavez, V. I. Descalzi, L. G. Podesta, and F. G. Villamil, "Addition of serum sodium into the MELD score predicts waiting list mortality better than MELD alone," Liver Transplantation, vol. 11, no. 3, pp. 336-343, 2005.

[18] J. Cai, K. Wang, T. Han, and H. Jiang, "Evaluation of prognostic values of inflammation-based makers in patients with $\mathrm{HBV}$ related acute-on-chronic liver failure," Medicine, vol. 97, no. 46, Article ID e13324, 2018.

[19] R. P. M. Te Dorsthorst, J. Hendrikse, M. T. Vervoorn, V. Y. H. van Weperen, and M. A. G. van der Heyden, "Review of case reports on hyperkalemia induced by dietary intake: not restricted to chronic kidney disease patients," European Journal of Clinical Nutrition, pp. 1-8, 2018.

[20] Y. Iwakiri and R. J. Groszmann, "The hyperdynamic circulation of chronic liver diseases: From the patient to the molecule," Hepatology, vol. 43, no. 2, pp. S121-S131, 2006.

[21] U. Udensi and P. Tchounwou, "Potassium homeostasis, oxidative stress, and human disease," International Journal of Clinical and Experimental Physiology, vol. 4, no. 3, pp. 111-122, 2017.

[22] S. S. Sheen, R. W. Park, D. Yoon, G.-T. Shin, H. Kim, and I.-W. Park, "The Model for End-stage Liver Disease score is potentially a useful predictor of hyperkalemia occurrence among hospitalized angiotensin receptor blocker users," Journal of Clinical Pharmacy and Therapeutics, vol. 40, no. 1, pp. 48-54, 2015. 


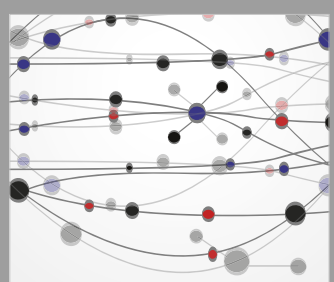

The Scientific World Journal
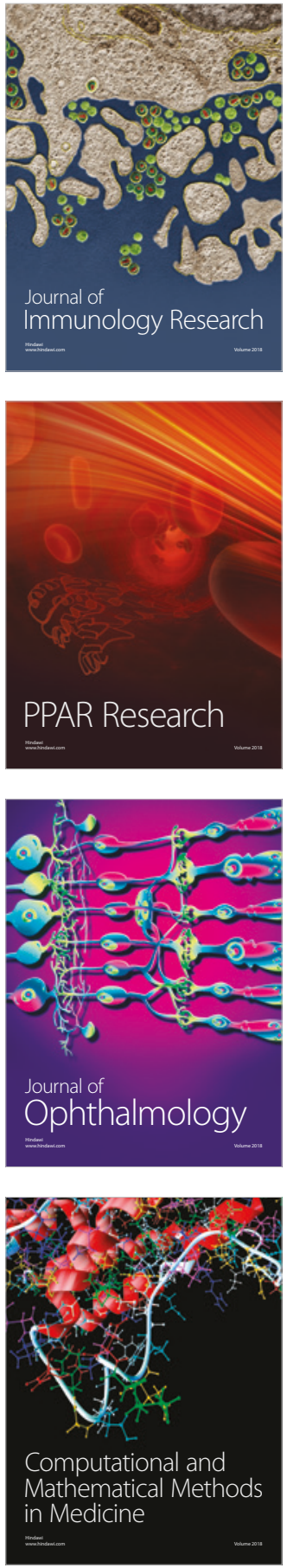

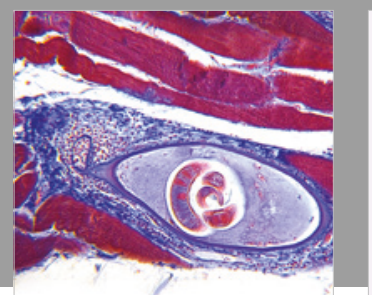

Gastroenterology Research and Practice

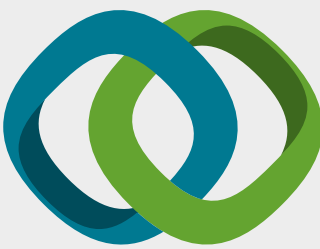

\section{Hindawi}

Submit your manuscripts at

www.hindawi.com
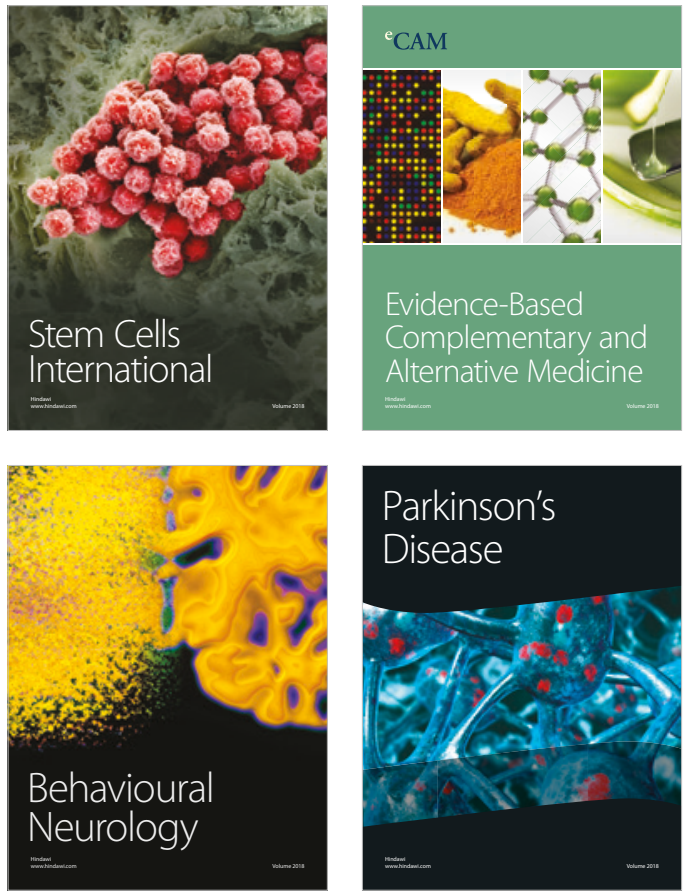

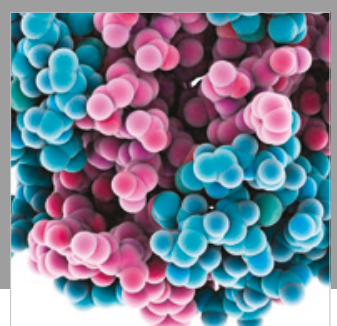

ournal of

Diabetes Research

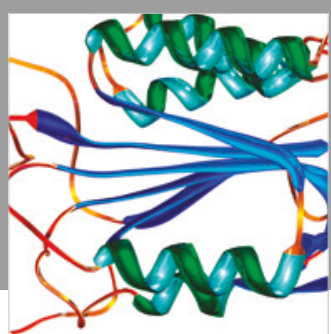

Disease Markers
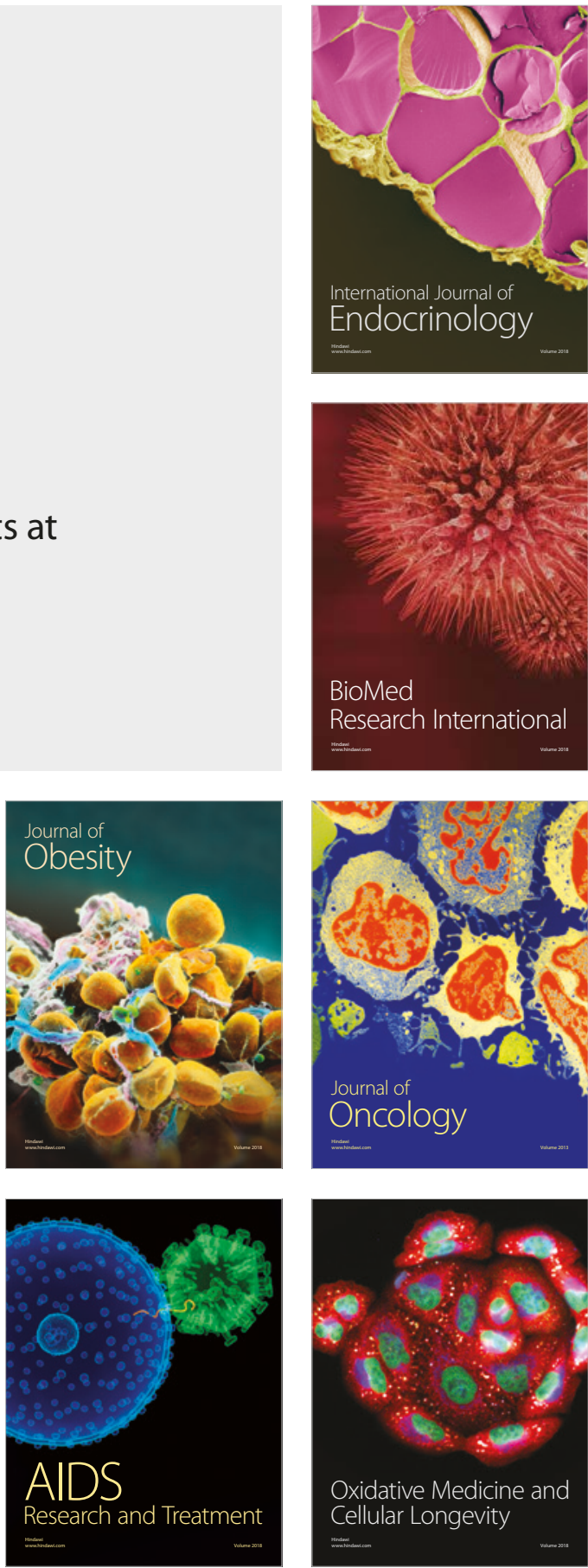